\title{
Order-disorder transition in nanoscopic semiconductor quantum rings
}

\author{
Peter Borrmann and Jens Harting \\ Department of Physics, Carl von Ossietzky University Oldenburg, D-26111 Oldenburg, Germany
}

(Dated: November 12, 2018)

\begin{abstract}
Using the path integral Monte Carlo technique we show that semiconductor quantum rings with up to six electrons exhibit a temperature, ring diameter, and particle number dependent transition between spin ordered and disordered Wigner crystals. Due to the small number of particles the transition extends over a broad temperature range and is clearly identifiable from the electron pair correlation functions.
\end{abstract}

PACS numbers: 73.23.-b,73.20.Dx,31.15.Kb,71.45.Lr

Nanoscopic semiconductor quantum rings (QRs), which recently have been experimentally realized by Lorke et. al [1], are next to quantum wires probably the best prototypes of quasi one dimensional quantum systems. QRs can be viewed as rotating Wigner crystals with promising features for application in microelectronics. They can be modeled using a simple Hamiltonian of the form

$$
H=\sum_{i=1}^{n}\left(\frac{\mathbf{p}_{i}^{2}}{2 m^{*}}+\frac{1}{2} m^{*} \omega_{0}^{2}\left(r_{0}-r_{i}\right)^{2}\right)+\sum_{i<j} \frac{e^{2}}{\kappa r_{i j}},
$$

where $\kappa=12.9$ and $m^{*}=0.067 m_{e}$ are the material constants of GaAs [2] [12]. The parameter $r_{0}$ is the radius of the quantum ring and $\omega_{0}$ defines the strength of the two-dimensional potential [1, 3]. Fig. 11(a) displays the shape of the ring potential. QRs can be tuned from quasi one-dimensional to two-dimensional systems by variation of the ring diameter and the potential strength.
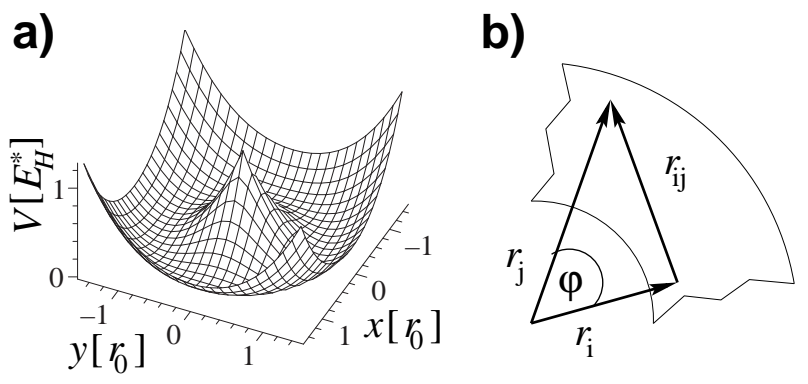

FIG. 1: Potential energy surface of a semiconductor quantum ring (a) and schematic illustration of the definition of $\varphi(b)$.

While mesoscopic QRs have been investigated theoretically and experimentally in depth [4], nanoscopic rings with strong quantum effects are of increasing interest. Koskinnen et al. [5] reported CI calculations of rotational and vibrational excitations of nanoscopic QRs with up to $N=7$ electrons. They claim that QRs behave like rather rigid molecules or Wigner crystals with antiferromagnetic order in the ground state. Ahn et al. [6] considered stacked nanoscopic rings and found an $N$ - dependent Stark effect. Experimentally it has been found that the emission energies of QRs change abruptly whenever adding an electron [7].
In this letter we present the results of path integral Monte Carlo (PIMC) simulations of single nanoscopic QRs with up to 8 electrons and different radii $r_{0}$. We show that they undergo a temperature, radius and particle number dependent spin order-disorder transition. Furthermore, the influence of quantum effects on the spatial electron distribution as well as the addition energies $\Delta E$ are given. Our results for the addition energies, i.e. the energy that is needed to place an additional electron in a ring, is compared to the experimental results of Warburton et al. [7].

In contrast to Hartree-Fock and spin density functional theory (DFT) PIMC samples without any approximation the full many body wavefunction instead of single or sums of Slater determinants. Especially for highly correlated electron systems this is a major advantage of PIMC. Another benefit of PIMC is the possibility to study temperature dependent phenomena. For quantum dots the problems of different density functional approaches have intensively been discussed in the past [2, 8, 9, 11. The so called fermion sign problem is still a topic of actual research and restricts the application of PIMC to a limited number of fermions and for QRs to a temperature of at least $10 \mathrm{~K}$.

The Feynman path integral for an $N$-electron system with position eigenket $\left|\mathbf{r}_{i}, s_{i}\right\rangle\left(s_{i}= \pm \frac{1}{2}\right.$ for spin-up and spin-down electrons) in an external potential can be rewritten as 10

$$
\begin{aligned}
Z & =\int\left[\prod_{\gamma=1}^{M} \prod_{i=1}^{N} \mathrm{~d} \mathbf{r}_{i}(\gamma)\right] \prod_{\delta=1}^{M} \operatorname{det}(A(\delta, \delta+1)) \\
& \times \exp \left(-\frac{\beta}{M} \sum_{\alpha=1}^{M} V\left(\mathbf{r}_{1}(\alpha), \ldots, \mathbf{r}_{N}(\alpha)\right)\right)+\mathcal{O}\left(\frac{\beta^{3}}{M^{2}}\right)
\end{aligned}
$$

with

$$
\begin{aligned}
& (A(\alpha, \alpha+1))_{i, j} \\
& =\left\{\begin{array}{lll}
\left\langle\mathbf{r}_{i}(\alpha)\left|\exp \left(-\frac{\beta}{M} \frac{\mathbf{p}^{2}}{2 m}\right)\right| \mathbf{r}_{j}(\alpha+1)\right\rangle & : & s_{i}=s_{j} \\
0 & : & s_{j} \neq s_{j}
\end{array}\right.
\end{aligned}
$$

and the boundary condition $\mathbf{r}_{j}(M+1)=\mathbf{r}_{j}(1)$. For $M \rightarrow \infty$ eq. (2) becomes exact. Standard Metropolis 

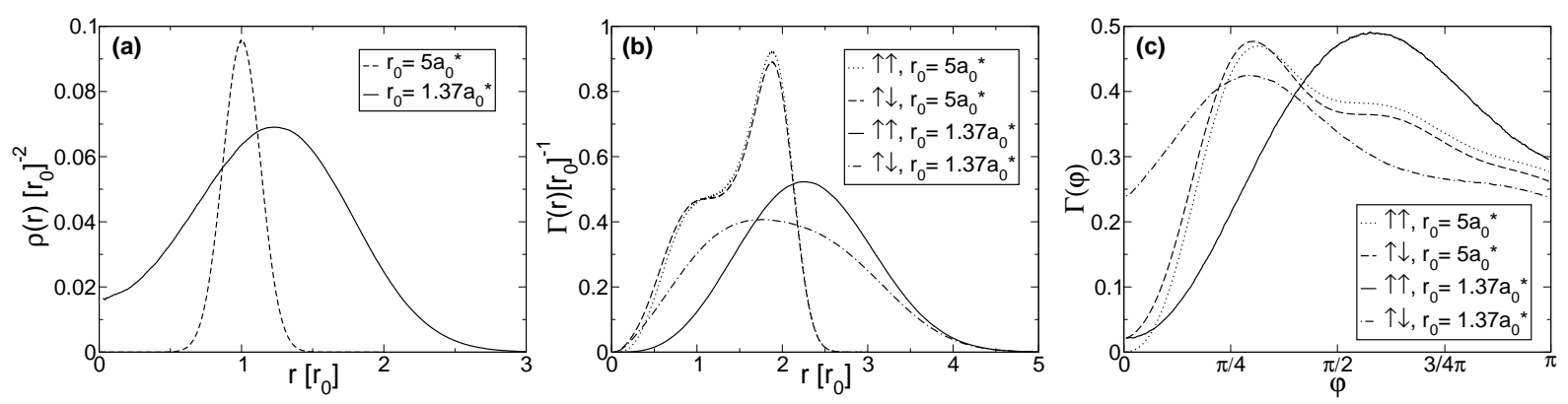

FIG. 2: Radial electron density (a), radial (b) and angular (c) pair correlation functions for $N=6, S=0$, and $r_{0}=1.37 a_{0}^{*}$ and $5 a_{0}^{*}$ at $T=15 \mathrm{~K}$.

Monte Carlo (MC) techniques can be utilized to evaluate the integral in (2).

The basic quantities reflecting the spatial structure of the electron configuration are the electronelectron (distance) pair correlation functions $\Gamma_{i, j}(r)=$ $\left\langle\delta\left(r-\left|\mathbf{r}_{i j}\right|\right)\right\rangle$, the angular pair correlation functions $\Gamma_{i, j}(\varphi)=\left\langle\delta\left(\varphi-\left|\varphi_{i}-\varphi_{j}\right|\right)\right\rangle$, and the radial electron density $\rho_{i}(r)=\frac{1}{2 \pi r}\left\langle\delta\left(r-\left|\mathbf{r}_{i}\right|\right)\right\rangle$, from which all energies can be calculated using the Hypervirial theorem (for details of our method see (9]). The definitions of $\varphi$ and $\mathbf{r}_{i j}$ are illustrated in Fig. I (b). To take the particle symmetry into account we introduce $\Gamma_{i j}=\Gamma^{\uparrow \downarrow}$ for $s_{i} \neq s_{j}, \Gamma_{i j}=\Gamma^{\uparrow \uparrow}$ for $s_{i}=s_{j}=\frac{1}{2}$, and $\Gamma_{i j}=\Gamma^{\downarrow \downarrow}$ for $s_{i}=s_{j}=-\frac{1}{2}$, respectively. Obviously for $S=0$ we have $\Gamma^{\downarrow \downarrow}=\Gamma^{\uparrow \uparrow}$.

In our simulations we controlled the systmatic error arising from the limited number of timeslices $M$ and the statistical MC error carefully. By choosing $M \times T \simeq 600$ and using up to 10 billion $\left(10^{10}\right)$ MC steps per run we pushed the overall error of all energy expectation values below $0.3 \%$. Our Fortran-code is completely parallelized using MPI and Lapack and most calculations have been performed on a Cray T3E with 64 processors.

We fixed the strength of the harmonic potential $\hbar \omega_{0}=$ $12 \mathrm{meV}$, resulting in effective atomic units for the Hartree energy $E_{H}^{*}=10.995 \mathrm{meV}$ and the Bohr radius $a_{0}^{*}=$ $10.1886 \mathrm{~nm}$. A ring diameter $r_{0}=14 \mathrm{~nm}=1.37 a_{0}^{*}$ than corresponds to the experimental setup of Lorke et al.. To investigate the ring size dependence we performed additional calculations for $r_{0}=50.94 \mathrm{~nm}=5 a_{0}^{*}$.

Fig. 2(b) and (c) display the pair correlation functions of QRs with $N=6, S=0$ and ring diameters $r_{0}=1.37 a_{0}^{*}$ and $5.0 a_{0}^{*}$ at $T=15 \mathrm{~K}$. As expected for both diameters peaks at $\varphi \approx \pi / 3$ and $\varphi \approx 2 \pi / 3$ occur in $\Gamma(\varphi)$. For $r_{0}=5 a_{0}^{*}$ the angular pair correlation functions for electrons with equal and unequal spin are almost identical, i.e. the Pauli principle does not play an important role in this case. In contrast, for $r_{0}=1.37 a_{0}^{*}$ the pair correlation functions show a strong spin dependence. The electrons arrange on the ring with anti-ferromagnetic order. Such spin density waves have been predicted by Koskinen et al. [5, 11]. The role of quantum effects is reflected as well in Fig. 22 (a). For the smaller ring size the radial electron density is much broader and nonvanishing at the ring center, implying that the system does not behave like a quasi 1D-system. In a perfect hexagonal Wigner crystal the equilibrium distances of the electrons would be $r=1,1.73$, and $2.0 r_{0}$. For $r_{0}=5.0 a_{0}^{*}$ the distance pair correlation function is approximately a properly weighted superposition of Gaussians centered at these distances. For $r_{0}=1.37 a_{0}^{*}$ the pair correlation function depends on the total spin of a pair and is broadened up to $4 r_{0}$. From Fig. 2 (b) and (c) we infer that the most probable configuration is one where the electrons are ordered on a zig-zag line around the circle, i.e. the electrons arrange alternately in the inner and the outer part of the ring.

Fig. 3 displays the angular spin density pair correlation functions for both ring diameters, temperatures $T=15,30$, and $90 \mathrm{~K}$ and particle numbers $N=4$, and 6 . In all cases the large angle correlations disappear with increasing temperature and at $T=90 \mathrm{~K}$ only at small angles a spin correlation is still visible. For $r_{0}=5.0 a_{0}^{*}$ the negative correlation at small angles increases with increasing temperature. However, this is simply due to the fact that the values of both correlation functions at small angles become larger with increasing temperature. The spin correlations for the small ring are about one order of magnitude larger than those in the ring with radius $r_{0}=5.0 a_{0}^{*}$ (Note the different scalings of the abscissae). A comparison between $N=4$ and $N=6$ shows that the spin correlation is smaller for the larger system. A probable reason for this is that for $N=6$ the contribution of the Coulomb repulsion is much larger (see Table II) freezing the electron in the Wigner crystal and - thinking in the picture of one-particle wavefunctions - making the overlap between one-particle wavefunctions smaller.

In summary, from Fig. 3 can be inferred that a spin order-disorder transition appears with increasing temperature, increasing electron number and increasing ring size.

Next we consider the temperature dependence of the spin order-disorder transition in some more detail. Fig. A displays the mean values of the angular separation of the electrons with equal and unequal spin for $N=4$ and 6 , 


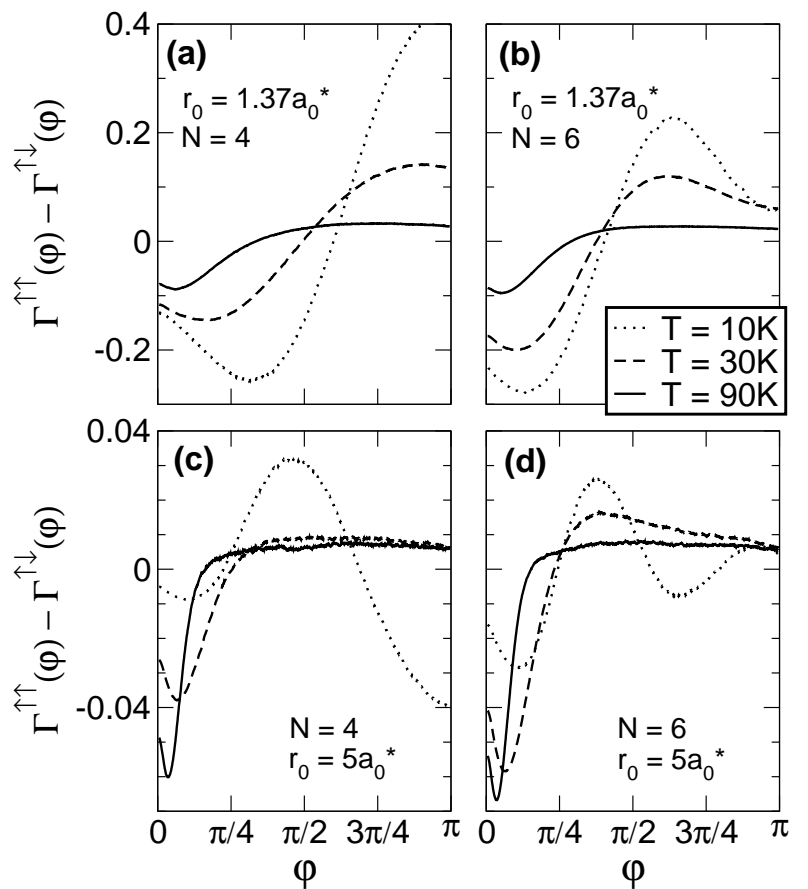

FIG. 3: Angular spin density correlation function $\left(\Gamma^{\uparrow \uparrow}(\varphi)-\right.$ $\left.\Gamma^{\uparrow \downarrow}(\varphi)\right)$ for $T=10,30$, and $90 \mathrm{~K}$ and (a) $N=4, S=0$, and $r_{0}=1.37 a_{0}^{*}$, (b) $N=6, S=0$, and $r_{0}=1.37 a_{0}^{*}$, (c) $N=4$, $S=0$, and $r_{0}=5 a_{0}^{*}$, and (c) $N=6, S=0$, and $r_{0}=5 a_{0}^{*}$.

$S=0$, and $r_{0}=1.37 a_{0}^{*}$ as a function of temperature. As expected from the results presented above the overall differences between the expectation values for equal and unequal spins approach zero with increasing temperature, i.e. the Pauli principle becomes less important. In addition, the values for $N=6$ are smaller than those for $N=4$. Obviously, this is because the available portion per particle of the ring volume is smaller for a larger number of electrons and the Coulomb repulsion is unable to disperse the particles. At $T=10 \mathrm{~K}$ and $N=4$ the contribution of the Coulomb term to the total potential energy is $53 \%$ for $r_{0}=1.37 a_{0}^{*}$ and only $36 \%$ for $r_{0}=5 a_{0}^{*}$, while for $N=6$ the difference between the different diameters is with $60 \%$ and $51 \%$ substantially smaller (see Table [i]).

The slope of $\left\langle\varphi^{\uparrow \downarrow}\right\rangle$ for $N=4$ can be understood as follows. Up to $40 \mathrm{~K}\left\langle\varphi^{\uparrow \downarrow}\right\rangle$ increases due spin disordering. At higher temperatures $\left\langle\varphi^{\uparrow \downarrow}\right\rangle$ decreases due to increasing thermal fluctuations.

Finally, we calculated the second energy differences $\Delta E=E_{N+1}-2 E_{N}+E_{N-1}$ also called addition energies, which are an indicator of the stability of a quantum ring with a given number of electrons. For quantum dots it was claimed that the electron configurations are given by Hunds rule [11] and consequently magic numbers occur at $N=2,6,12$, and 20 . Here we calculated only the addition energy up to $N=7$ for $25 \mathrm{~K}$ and $N=5$ for $10 \mathrm{~K}$. As expected due to the Pauli principle and the

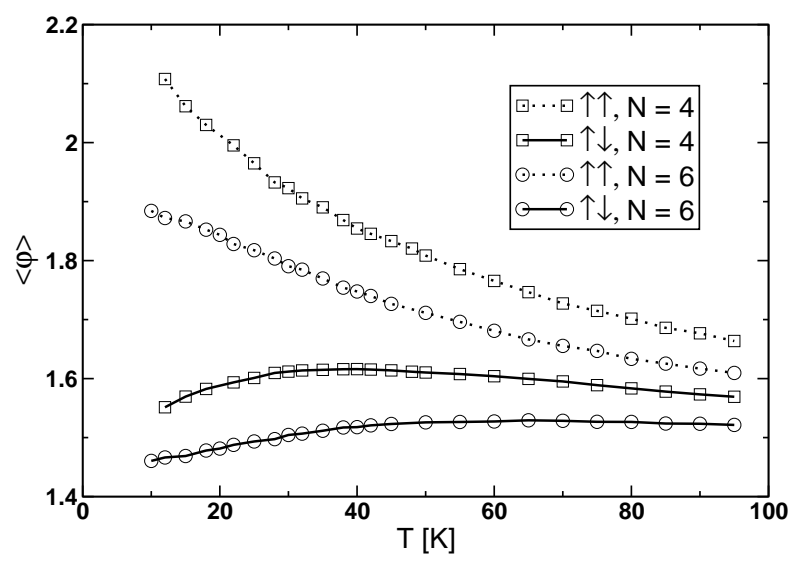

FIG. 4: Temperature dependence of the mean angles $\left\langle\phi^{\uparrow \uparrow}\right\rangle$ and $\left\langle\phi^{\uparrow \downarrow}\right\rangle$ for $N=4$ and $6, S=0$ and $r_{0}=1.37 a_{0}^{*}$.

Wigner crystal structure a strong odd even effect occurs. The general behavior of this effect does not change for $25 \mathrm{~K}$. However, for higher temperatures significant differences can be expected (see above). Warburton et al. [7] argued that the general features of shell effects occurring in QRs are the same as in quantum dots. As can be inferred from Fig. 5, our calculations confirm this for $N=6$. Furthermore, the addition energies are in the same range as those from photoluminescence measurements at $4 \mathrm{~K}[7]$. Due to the strong Coulomb repulsion in QRs, which grows with increasing electron number, it can be expected that shell effects become less important with increasing particle number.

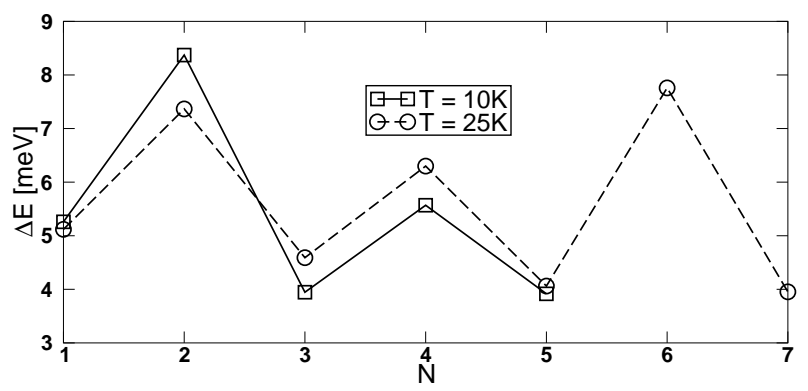

FIG. 5: Addition energies $\Delta E$ at temperatures $T=10$ and $25 \mathrm{~K}$ for $r_{0}=1.37 a_{0}^{*}$.

The effects described above are reflected quantitatively in Table 1 presenting the total energy $\left(E_{\text {tot }}\right)$, kinetic en$\operatorname{ergy}\left(E_{\text {kin }}\right)$, total potential energy $\left(E_{\text {pot }}\right)$, the energy due to the ring potential $\left(E_{\text {ring }}\right)$, and the Coulomb energy $\left(E_{\mathrm{c}}\right)$.

In conclusion, we presented the results of full many body wavefunction calculations for QRs with up to eight electrons. We found that the properties of the rings depend in an intriguing manner on the ring diameter, the particle number and the temperature, which in turn is due to spin correlation, Coulomb ordering and the gen- 
TABLE I: Total-, kinetic-, potential-, ring-, and Coulombenergies for $N=4$ and $6, S=0, r_{0}=1.37$ and $5 a_{0}^{*}$ for different temperatures. All energies are given in $\mathrm{meV}$.

\begin{tabular}{|c|c|c|c|c|c|c|c|}
\hline$N$ & $r_{0}\left[a_{0}^{*}\right]$ & $T[\mathrm{~K}]$ & $E_{\text {tot }}$ & $E_{\text {kin }}$ & $E_{\text {pot }}$ & $E_{\text {ring }}$ & $E_{\mathrm{c}}$ \\
\hline \multirow[t]{6}{*}{4} & 1.37 & 10 & 57.2 & 23.0 & 34.2 & 16.1 & 18.1 \\
\hline & & 25 & 59.3 & 24.4 & 35.0 & 16.8 & 18.2 \\
\hline & & 90 & 85.1 & 40.6 & 44.4 & 26.5 & 17.9 \\
\hline & 5 & 10 & 32.7 & 15.0 & 17.7 & 11.4 & 6.3 \\
\hline & & 25 & 36.1 & 17.7 & 18.4 & 11.6 & 6.8 \\
\hline & & 90 & 62.0 & 35.4 & 26.6 & 18.5 & 8.1 \\
\hline \multirow[t]{6}{*}{6} & 1.37 & 10 & 117.8 & 39.4 & 78.4 & 31.1 & 47.3 \\
\hline & & 25 & 122.5 & 42.6 & 79.9 & 32.9 & 46.9 \\
\hline & & 90 & 160.9 & 66.7 & 94.2 & 48.4 & 45.8 \\
\hline & 5 & 10 & 61.0 & 25.3 & 35.7 & 17.3 & 18.3 \\
\hline & & 25 & 65.7 & 28.8 & 36.9 & 17.8 & 19.1 \\
\hline & & 90 & 105.8 & 55.3 & 50.6 & 29.1 & 21.5 \\
\hline
\end{tabular}

eral strength of quantum effects. QRs exhibit a parameter dependent spin order-disorder transition. By variation of the ring diameter the system can be tuned from a quasi 1D Wigner crystal to a 2D structure. The accesible parameter ranges can be used to tune the properties of quantum rings to desired values. Because of the ring diameter as an additional parameter this qualifies them as even better candidates than quantum dots for possible applications in microelectronics. The addition energies calculated using PIMC are in good agreement with the experimental results of Warburton et al. 77 and reflect the predicted shell effects.

We wish to thank the Konrad Zuse Institut Berlin and the Regionales Rechenzentrum Niedersachsen for their excellent computer support and Oliver Mülken, Heinrich
Stamerjohanns and E. R. Hilf for fruitful discussions.

[1] A. Lorke, R. Luyken, A. Govorov, J. Kotthaus, J. Garcia, and P. Petroff, Phys. Rev. Lett. 84(10), 2223 (2000).

[2] K. Hirose and N. Wingreen, Phys. Rev. B 59 (4604) (1999).

[3] T. Chakraborty and P. Pietiläinen, Phys. Rev. B 50(12) (1994).

[4] L. Wendler and V. Fomin, Phys. Stat. Sol. B 191, 409 (1995). L. Wendler, V. Fomin, A. Chaplik, and A. Govorov, Phys. Rev. B 54(7), 4794 (1996). D. Mailly, C. Chapelier, and A. Benoit, Phys. Rev. Lett. 70(13), 2020 (1993).

[5] M. Koskinen, M. Manninen, B. Mottelson, and S. Reimann, arXiv:cond-mat 004095 (2000).

[6] K. Ahn and P. Fulde, Phys. Rev. B 62(8), 4813 (2000).

[7] R. Warburton, C. Schäflein, D. Haft, F. Bickel, A. Lorke, K. Karrai, J. Garcia, W. Schoenfeld, and P. Petroff, Nature 405, 926 (2000).

[8] C. Yannouleas, and U. Landman, Phys. Rev. Lett. 82 (5325) (1999).

[9] J. Harting, O. Mülken, and P. Borrmann, Phys. Rev. B 62(15),10207 (2000).

[10] M. Takahashi and M. Imada, J. Phys. Soc. Jpn. 53(3), 936 (1984). M. Takahashi and M. Imada, J. Phys. Soc. Jpn. 53(11), 3765 (1984). P. Borrmann and E. Hilf, Z. Phys. D 26, S350 (1993).

[11] M. Koskinen, M. Manninen, and S. Reimann, Phys. Rev. Lett. 79(7), 1389 (1997).

[12] The value of $\kappa=12.9$ for GaAs is slightly different from the for the experiments of Lorke et al. more appropiate value of $\kappa=14.5$ for InAs. We have chosen this value for easier comparison to previous quantum dot calculations. 